\title{
Sarcoglycanopathies: A clinico-pathological study
}

\author{
A. K. Meena, D. Sreenivas, C. Sundaram*, R. Rajasekhar\#, J. S. Sita, R. Borgohain, A. Suvarna, \\ S. Kaul \\ Departments of Neurology, *Pathology and ${ }^{\# C a r d i o l o g y, ~ N i z a m ' s ~ I n s t i t u t e ~ o f ~ M e d i c a l ~ S c i e n c e s, ~ P u n j a g u t t a, ~ H y d e r a b a d, ~ I n d i a ~}$
}

\begin{abstract}
Background: Limb girdle muscular dystrophy (LGMD) is a heterogeneous group of disorders characterized by limb girdle weakness. There are no clear clinical features that distinguish various types of LGMD. Materials and Methods: We studied 26 patients with chronic progressive weakness in limb girdle distribution without early facial involvement with muscle biopsies suggestive of dystrophy/myopathy and positive for dystrophin antibodies.
\end{abstract} Immunohistochemistry studies of muscle biopsies were done on all patients to classify different types of sarcoglycanopathies. Results: The mean age of presentation was in the third decade. There were 14 male and 12 female patients. The common pattern of inheritance was autosomal recessive, seen in $53.8 \%$. The more frequent type of LGMD was sarcoglycanopathy (SGP) (53.8\%). Amongst the SGPs, alpha-SGP (26.9\%) was the most common followed by beta-SGP (15.3\%), gamma-SGP (3.8\%) and delta-SGP (7.6\%). Calf hypertrophy was noted in $53.5 \%$ of LGMD and $57.1 \%$ of SGPs, extensor digitorum brevis hypertrophy in $42 \%$ of LGMD and $35.7 \%$ of SGPs, winging of scapula in $39.2 \%$ of the LGMD group and $35.7 \%$ of the SGPs, valley sign in $28.5 \%$ of the LGMD group and $21.4 \%$ of the SGPs. Hip abductor sign was positive in $71.4 \%$ of LGMD and $64.2 \%$ of SGPs. Differential weakness of knee flexors was more common in SGP $(57.1 \%)$. The mean creatine phosphokinase (CK) value was $2519 \mathrm{IU} / \mathrm{L}$ and was elevated in $92.8 \%$ patients. Muscle biopsy showed a dystrophic pattern in 75\% of LGMD and a myopathic pattern in the remaining. Symptomatic cardiac involvement was seen in one patient. ECG changes were seen in $44 \%$ of LGMD patients and $50 \%$ of the SGP. The common changes noted were T wave inversion in V1, V2 (16\%), left ventricular hypertrophy LVH (12\%) and right bundle branch block (RBBB) in $12 \%$ of the LGMD group. Conclusion: Sarcoglycanopathy is a more frequent form of LGMD whereas alpha type is the most common among the SGP. The four types of SGP do not differ in the pattern of muscle involvement. A relatively earlier onset, selective weakness of knee flexors and a very high CK may help differentiate
SGP from other forms of LGMD. Immunohistochemistry is very useful in classifying the different types of LGMD prior to genetic analysis.

Key words: Limb girdle muscular dystrophies, nonsarcoglycanopathy, sarcoglycanopathy

Limb girdle muscular dystrophies (LGMD) constitute a heterogeneous group of disorders with predominant limb girdle wasting and weakness with facial muscle sparing. Walton and Nattrass separated fascioscapulohumeral muscular dystrophy and grouped the pelvic atrophic form of Leyden and Mobius, the juvenile scapulohumeral form of Erb and the late onset forms of Nevi (1936) under LGMD. ${ }^{[1]}$ Therefore there was no clear characterization of the disorder. Limb girdle muscular dystrophies have been completely redefined following inputs from immunohistochemistry and molecular biology, which have made it possible to determine the genetic basis of the various disorders within the group.

LGMD are inherited as either autosomal recessive (AR), autosomal dominant (AD) or sporadic. The group of dystrophies is continuing to expand and presently there are 19 known forms of LGMD: seven types of AD (LGMD1A to LGMD1G) and 13 AR LGMD (LGMD2A to LGMD2M) ${ }^{[2]}$ Sarcoglycanopathies (SGs) are defined by the involvement of the dystrophin-glycoprotein complex (DGC) in the muscle fiber membrane, while the complex is normal in other forms of $\mathrm{AR}$ and $\mathrm{AD}$ LGMDs. ${ }^{[3]}$ The prevalence of LGMD seems to be extremely variable among populations depending on historical, geographical and cultural factors. Most of the literature relates to the Caucasian population. Reports from India on LGMDs and their subtypes are few, with only two studies published so far, one each from the west and the north of India. ${ }^{[4,5]}$

The aim of the study was to analyze the clinical presentation, phenotypic characteristics, inheritance pattern, biochemical, electrophysiological, histological, 
immunohistochemical characteristics and cardiac involvement in patients with limb girdle muscular dystrophy.

\section{Materials and Methods}

All patients presenting with predominant limb girdle weakness to the neuromuscular clinic between January 2002 and May 2004 at a tertiary care hospital-Nizam's Institute of Medical Sciences - in south India were studied. The inclusion criteria were: presence of chronic progressive limb girdle weakness with onset in pelvic girdle without early facial weakness, muscle biopsy compatible with dystrophy/myopathy and positive dystrophin on immunohistochemistry. The exclusion criteria were: congenital onset weakness of limb girdle muscles and onset in distal or facial muscles. A detailed pedigree chart was drawn based on which the inheritance pattern was determined. Autosomal recessive inheritance was considered if the patient had an affected sibling with horizontal transmission of the disease or where the parents had a history of consanguinity. Autosomal dominant inheritance was considered if vertical transmission was present with an affected family member in each generation. The patients not fitting into either recessive or dominant type were termed as sporadic.

The patients and their affected family members were clinically examined. Selective wasting, hypertrophy and contractures were noted with a special focus on the presence of the valley $\operatorname{sign}^{[6]}$ (a linear or oval depression (due to wasting) of the posterior axillary fold with hypertrophied or preserved muscles on two borders (i.e., the infraspinatus inferomedially and deltoid superolaterally), with the appearance of a valley between the two mounts) or scapular winging and hip abductor sign (the selective and differential weakness between weak hip adductors and stronger hip abductors results in the hip abduction sign, which becomes apparent while getting up from the sitting posture). ${ }^{[4]}$ The patients were also examined for the presence of selective muscle weakness of agonists or antagonists across the elbow, the knee and the ankle joints. Power grading of all major individual muscles was done on the basis of the Medical Research Council grading for power examination. Respiratory muscle involvement was assessed clinically by performing single breath count.

All subjects underwent a complete biochemical evaluation including creatine phosphokinase (CK) and thyroid hormones. Nerve conduction studies and electromyography (EMG) were performed on all patients. Spontaneous activity, motor unit amplitude, duration, interference pattern and amplitude and velocities of sensory and motor nerves were noted. Muscle biopsy was performed on all patients by using the standard open biopsy technique under local anesthesia. The biopsy specimens were snap-frozen in chilled isopentane for frozen sections. Histochemical stains were done with ATPase (at pH 9.4,4.6 and 4.3), nicotinamide adenine dehydrogenase (NADH), succinate dehydrogenase (SDH), masson trichrome, modified Gomori trichrome (MGT) and further special stains wherever necessary.

The biopsy specimens were initially immunostained for dystrophin and were further stained with sarcoglycan antibodies. Dystrophin staining was done using a mouse monoclonal antibody, IgG1 (NCL-DYS2) that reacts against the carboxy terminus (between amino acids 3669 and 3685 ) of the dystrophin protein. The alpha-SG antibody is a mouse monoclonal antibody of IgG1 (NCL-a-SARC) against adhalin and belongs to the clone of Ad1/20A6 [Figure 1]. The beta-SG (bSarc/5B1), gamma-SG (35DAG/ 21B5), delta-SG (d-Sarc3/12c1) antibodies are all mouse monoclonal antibodies of the IgG1 group. ${ }^{[7]}$ Immunostaining with merosin was done with biopsy specimens from three patients with onset below five years of age to exclude merosin-negative congenital muscular dystrophy. Complete absence of a particular type of sarcoglycan was considered to be a primary defect while interpreting the results.

All patients underwent cardiac evaluation including electrocardiography (ECG) and 2D-echocardiography. Thirty age-matched controls also underwent cardiac evaluation for comparison. The MRI of the thigh muscles was performed in two patients using a 1.5T MRI machine.

\section{Results}

Clinical data: Twenty-six patients were included in the study (14 male and 12 female). Age at presentation ranged from 14-46 years (mean-28 \pm 8.9 yrs). The duration of disease ranged from 1-35 years (mean-6.8 \pm 8.3 yrs). Mean age of onset of the disease was 21-10.8 years [Table 1].

In four patients the onset of the disease was in the first decade, nine in the second, 11 in the third, three in the fourth and in one patient the onset of the disease was in the fifth decade. All patients were born in Andhra Pradesh and had their ancestral roots in this state. The pattern of inheritance was as follows: autosomal recessive in 14 patients; autosomal dominant in one patient and sporadic in 11 patients. Five patients showed similar patterns of

\begin{tabular}{ll}
\hline \multicolumn{2}{l}{ Table 1: Demographic and clinical profile of LGMD patients } \\
\hline & LGMD patients $(\mathbf{n = 2 6})$ \\
Mean age of presentation (years) & $28 \pm 8.9$ \\
Mean age of onset (years) & $21 \pm 10.8$ \\
Mean disease duration (years) & $6.8 \pm 8.3$ \\
Inheritance & $\mathrm{AR}-53.8 \%, \mathrm{~S}-42.3 \%, \mathrm{AD}-3.8 \%$ \\
Calf hypertrophy & $53.8 \%$ \\
EDB hypertrophy & $38.4 \%$ \\
Valley sign & $34.6 \%$ \\
Scapular winging & $38.4 \%$ \\
Hip abductor sign & $69.2 \%$ \\
Mean CK (U) & $2553.3 \pm 2903.6$ \\
Muscle biopsy & Dystrophic-76.9\%, \\
& Myopathic- $22.2 \%$ \\
\hline
\end{tabular}


weakness in the family.

Wasting involving the proximal pelvic girdle muscles and thigh muscles was seen in 20 patients whereas seven of these also had wasting of the shoulder girdle muscles. The muscles that were predominantly wasted included deltoid, supraspinatus, infraspinatus, trapezius, rhomboides and paraspinal muscles. Wasting in the lower limbs predominantly involved the quadriceps and the adductor group of thigh muscles. The shoulder girdle weakness was present in 22 patients in addition to the pelvic girdle weakness. Six patients had weakness of hand muscles while nine patients suffered from distal leg weakness. The elbow flexors were weaker than the extensors in four patients. The extensors were weaker than the flexors at the elbow joint in four patients. The knee flexors were weaker than the extensors in nine patients whereas the converse was true in two patients. Mild facial weakness was present in two patients both of whom had disabling myopathy. None of the patients had contractures. Clinically, respiratory muscle involvement was not seen in any of the patients. Calf hypertrophy was present in $53.5 \%$ of LGMD and $57.1 \%$ of SGs. Extensor digitorum brevis (EDB) hypertrophy was present in $42 \%$ of the LGMD and $35.7 \%$ of the SGs. The CK was elevated (>200IU/L) in 24 patients and CK levels above 2000IU/L (10 times the normal) were seen in 13 patients.

Out of the 26 patients, 24 underwent detailed cardiac evaluation and 12 of these showed normal ECG. The common ECG change was T wave inversion from V1 to V3, seen in four patients, left ventricular hypertrophy and RBBB (right bundle branch block) pattern in three patients each and poor $\mathrm{R}$ wave progression from $\mathrm{V} 1$ to $\mathrm{V} 3$ and $\mathrm{R}>\mathrm{S}$ in $\mathrm{V} 1$ and V2 in two patients each. Left atrial hemi-block and Tall $\mathrm{T}$ waves in $\mathrm{V} 1$ and $\mathrm{V} 2$ were seen in one patient each. 2D-Echo was normal in 18 patients. Trivial mitral regurgitation was observed in two patients and trivial tricuspid regurgitation in three. One patient had a dilated left ventricle with poor ejection fraction while another had diastolic dysfunction and yet another had left atrial enlargement.

Common abnormality in muscle biopsy was a dystrophic pattern, seen in 20 patients, followed by myopathic changes seen in another six [Table 2]. Histological features in sarcoglycanopathy (SGP) in the present study and the
Table 2: Histological characteristics in patients with Limb girdle muscular dystrophy ${ }^{[4]}$

\section{Muscle biopsy findings}

Fiber size variation

Central nuclei

Rounded fibers

Necrosis

Split fibers

Increased connective tissue

Atrophic fibers

Hypertrophic fibers

Angulated fibers

Lymphocytic infiltration
Number of patients (\%)

$25 / 26(96.1)$

$14 / 26(53.8)$

$13 / 26(50)$

$14 / 26(53.8)$

$11 / 26(42.3)$

$13 / 26(50)$

$13 / 26(50)$

$1 / 26(3.8)$

$3 / 26(11.5)$
$5 / 26(19.2)$

Table 3: Comparison of biopsy findings in patients with SGPs in our series and Khadilkar series ${ }^{[4]}$

\begin{tabular}{|c|c|c|}
\hline Fiber size variation & $14 / 14(100)$ & $96 \%$ \\
\hline Central nuclei & $9 / 14(64.2)$ & $80 \%$ \\
\hline Rounded fiber & $6 / 14(42.8)$ & $72 \%$ \\
\hline Necrosis & $3 / 14(21.4)$ & $64 \%$ \\
\hline Split fibers & $6 / 14(42.8)$ & $40 \%$ \\
\hline Increased connective tissue & $6 / 14(42.8)$ & $40 \%$ \\
\hline Atrophic fibers & $5 / 14(35.7)$ & \\
\hline Hypertrophic fibers & $8 / 14(57.1)$ & $36 \%$ \\
\hline Angulated fibers & $1 / 14(0.7)$ & \\
\hline Lymphocytic infiltration & $2 / 14(1.42)$ & \\
\hline
\end{tabular}

Figures in parentheses are in percentage

earlier study by Khadilkar ${ }^{[4]}$ are shown in Table 3.

Sarcoglycan (SG) analysis was done in all patients. Four patients with onset in the first decade underwent immunostaining with merosin to exclude merosinnegative congenital myopathy and all were positive for merosin. The common subtype of the LGMD was sarcoglycanopathy group (53.8\%). Amongst the SGPs, alpha-SGP (26.9\%) was the most common [Figure 1] followed by the beta-SGP (15.3\%). The least common was the gamma-SGP (3.8\%) [Figure 2]. Comparative data between different subtypes of the SGPs is shown in Table 4. Clinical data obtained was also compared between the SGP and the non-SGP groups.

\section{Discussion}

In the present study, both sexes were found to be almost equally affected. The age of onset of symptoms was lower in males compared to females. The AR transmission is the most common inheritance pattern in our study

Table 4: Comparison between different subtypes of Sarcoglycanopathies

\begin{tabular}{|c|c|c|c|c|}
\hline & $\begin{array}{c}\text { Alpha-SG } \\
n=7\end{array}$ & $\begin{array}{c}\text { Beta-SG } \\
n=4\end{array}$ & $\underset{n=1}{\operatorname{Gamma-SG}}$ & $\begin{array}{c}\text { Delta-SG } \\
n=2\end{array}$ \\
\hline Mean age of presentation (years) & 29.7 & 21.7 & 30 & 24 \\
\hline Mean age of onset (years) & 22 & 15.2 & 23 & 20.5 \\
\hline Mean disease duration (years) & 7.7 & 5.25 & 7 & 3.5 \\
\hline Inheritance & AR-71.4\%, S-28.5\% & AR- $50 \%$ S- $50 \%$ & AR- $100 \%$ & AR- $50 \%$ S- $50 \%$ \\
\hline Calf hypertrophy & $57.1 \%$ & $100 \%$ & - & - \\
\hline EDB hypertrophy & $28.5 \%$ & $50 \%$ & - & $50 \%$ \\
\hline Valley sign & $28.5 \%$ & $25 \%$ & - & - \\
\hline Scapular winging & $57.1 \%$ & $25 \%$ & - & - \\
\hline Hip abductor sign & $71.4 \%$ & $50 \%$ & - & $100 \%$ \\
\hline Mean CK (U) & 2187 & 2603 & 2881 & 11,400 \\
\hline Muscle biopsy D-Dystrophic M-Myopathic & $D-86 \% M-14 \%$ & D- $50 \% M-50 \%$ & D- $100 \%$ & D-50\% M- $50 \%$ \\
\hline
\end{tabular}




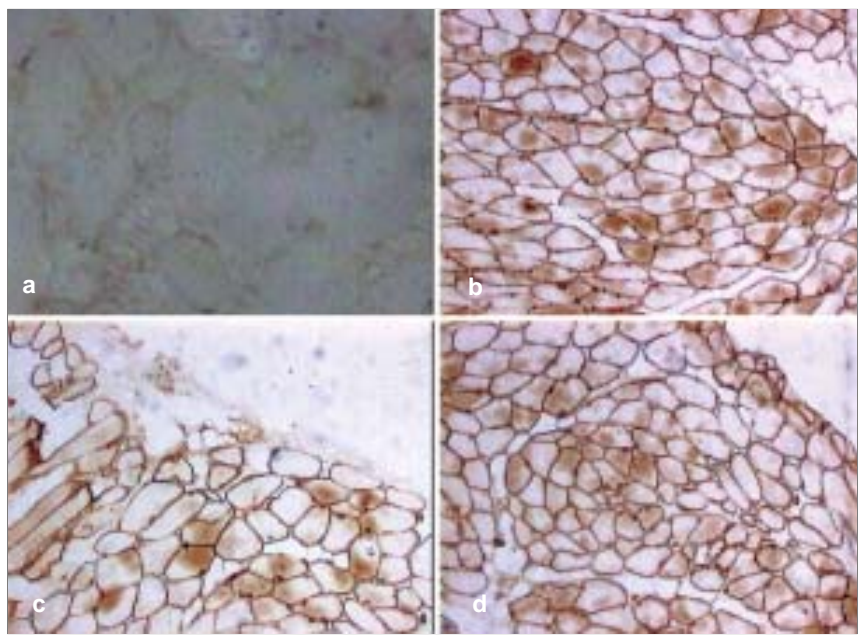

Figure 1: Muscle biopsy of a patient with alpha-sarcoglycanopathy, positive staining with antibodies against dystrophin, negative staining with antibodies against alpha-SG and positive staining for Beta , Gamma and Delta SG. (a) Alpha SG, (b) Beta SG, (c) Gamma SG, and (d) Delta SG
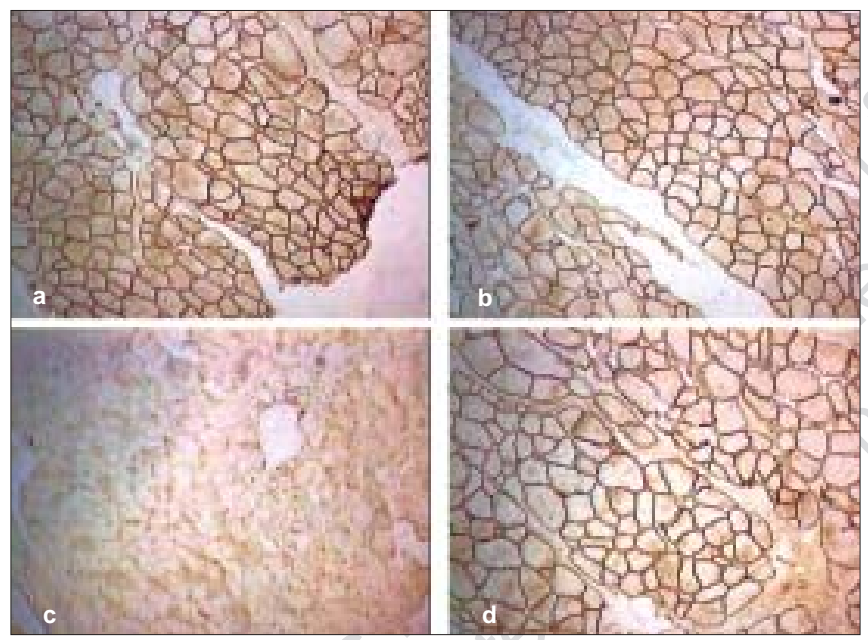

Figure 2: Muscle biopsy of a patient with gamma-sarcoglycanopathy, positive staining with antibodies against dystrophin and showing negative staining with antibodies against gamma-SG and staining positive for Alpha, Beta and Delta SG. (a) Alpha SG, (b) Beta SG, (c) Gamma SG, and (d) Delta SG

(53.8\%), similar to previous results..$^{[8,9]}$ The high incidence of recessive inheritance in our series may be because of the high rate of consanguineous marriages in the south Indian state of Andhra Pradesh.

Common muscles to be hypertrophied included the EDB and the calf muscles. The other muscles that were selectively hypertrophied were the deltoid, the brachioradialis and the quadriceps in one patient. This is comparable to the previous series. ${ }^{[8-12]}$ Muscle hypertrophy seen in both SGP and non-SGP does not help to differentiate among the various LGMDs. Selectivity of either agonists or antagonists across major joints is a feature of LGMD, especially at the hip. Abductor sign at the hip was present in $71.4 \%$ of the LGMD group and was more common in the non-SGPs (75\%) than the SGPs although the difference was not statistically significant.
Abductor weakness has been previously reported in childhood onset gamma SGPs and alpha SGPs in adults. ${ }^{[10,13]}$

In the series by Khadilkar, ${ }^{[4]}$ the hip abductor sign was seen in $64 \%$ of all SGPs and the winging of scapula in $44 \%$ of the cases. The differential weakness at the elbow was noted in $28.5 \%$ of the LGMD group with the elbow flexors weaker than the extensors. Differential weakness at the knee was noted in $39.2 \%$ of the LGMD group and $57.1 \%$ of the SGP group with $80 \%$ of these patients having weaker hamstrings than quadriceps, unlike in DMD. The valley sign is also seen in patients with LGMD but is less common in patients with sarcoglycanopathy. Selective weakness of the muscle groups at the knee and a very high CK was common in the SGP group. This difference in the presentation between the two groups may help distinguish SGPs from other LGMDs. Sarcoglycanopathy generally manifests earlier in life than other forms of LGMD.

The dystrophic pattern was the most common biopsy picture in the LGMD group (75\%). In the series by Khadilkar, ${ }^{[4]}$ the dystrophic pattern was seen in all the patients with SGs. Comparison between autosomal recessive and autosomal dominant was not possible as only one patient had AD inheritance. We found increased hypertrophic fibers, interstitial fibrosis and lobulated fibers in patients with a long history of muscle symptoms. Similar findings were observed in other studies as well. ${ }^{[7]}$

The SGPs constituted 53.8\% of the LGMD in the present study. In a series of 54 cases of LGMD by Khadilkar, the SGPs constituted $46.2 \%$ of the cases. Sharma et $a{ }^{[5]}$ on the other hand, reported a very low incidence of SGPs (11.8\% of LGMD). The high incidence in the present study and that by Khadilkar could be due to selection bias. Multiple sarcoglycan deficiency was noted in nine out of 12 patients (75\%). Multiple sarcoglycan deficiency was seen in five out six patients with alpha-SGs, all three patients with beta-SGs, one out of two patients with deltaSG and in one patient with gamma-SG. Khadilkar et al reported multiple sarcoglycan deficiencies in $84 \%$ of the patients. Primary beta- and delta-SG deficiency was seen in the remaining $16 \%$ of the cases. ${ }^{[4]}$ Dincer et $a l,{ }^{[14]}$ in another series involving 20 Turkish families found that the common subtype of LGMD was calpainopathy followed by an SG deficiency. Sarcoglycanopathies constituted 25\% of the AR LGMD in the series by Van der Kooi. ${ }^{[7,8]}$ The most common LGMDs in the United States are calpainopathies (12\%), dysferlinopathies (18\%), sarcoglycanopathies (15\%) and dystroglycano-pathies $(15 \%) .{ }^{[15]}$ In the present study immuno-histochemistry was not done to define all types of LGMDs, hence no definite observations can be made on the different types of LGMDs.

In our series, the alpha-SG constituted $25 \%$ of the total LGMD group and $25.9 \%$ of the AR/sporadic group. The frequency of the alpha-SG deficiency on the 
immunohistochemistry in the LGMD varies from 0 to $35 \%$ in the literature. ${ }^{[11,16]}$ Four patients had beta-sarcoglycan deficiency whereas two had delta and only onw had gamma deficiency. Interestingly, the gamma-SGP was the most common type in the study by Sharma et al, from the northern part of India. No clear clinical observations in relation to the pattern of muscle involvement distinguished the four types of SGPs in the present study, just like in the previous study, although milder forms are more associated with mutations in alpha-SG. ${ }^{[17]}$ The pattern of muscle involvement differs across the various types of LGMDs and so does the natural course.

In the present study, cardiac involvement was seen in $50 \%$ of the SGPs. There was no difference in the ECG changes and the cardiac abnormalities in the 2D-Echo between the two groups. None of the patients in the SG group had symptomatic cardiac or severe cardiac involvement in the 2D-Echo. Melacini et al studied 13 patients with SG mutation where the ECG or the 2DEcho changes were present in only $30 \%$ of the patients. ${ }^{[18]}$

There was no correlation between the cardiac involvement and the type of SG mutation and the former was never severe. Politano from Italy studied the cardiac involvement in 20 patients with an SG deficiency and found cardiomyopathy (CMP) in $43.7 \%$, arrhythmogenic CMP in $6.3 \%$ and signs of dilated CMP in $8.7 \%$ of the patients. ${ }^{[19]}$ Recent detailed clinical descriptions of the different subtypes of the LGMD mention the absence of cardiac abnormalities in the type $2 \mathrm{~B}^{[20]}$ and a high frequency of cardiac anomalies in the type 2C. ${ }^{[13]}$

\section{Conclusion}

LGMD are clinically a heterogeneous group of disorders commonly presenting in the third decade of life, with an equal male to female ratio. The common pattern of inheritance is the autosomal recessive. Clinically, selective limb girdle wasting and weakness (indicated by scapular winging, valley sign, and hip abductor sign) are characteristic of the limb girdle muscular dystrophies. The valley sign, selective weakness of hamstrings versus quadriceps and high CK values may help differentiate a sarcoglycanopathy from other types of LGMDs. Sarcoglycanopathy, whose most common type is the alphaSG, seems to be a more common type of LGMD. Also, immunohistochemistry is important in classifying the various forms of LGMD prior to genotypic diagnosis. Nonspecific abnormalities in ECG were also common. Symptomatic cardiac involvement was uncommon, though 2D-Echo showed subclinical abnormalities.

\section{References}

treatment of the myopathies. Brain 1954;77:169-231.

2. Daniele N, Richard I, Bartoli M. Ins and outs of therapy in limb girdle muscular dystrophies. Int J Biochem Cell Biol 2007;Epub print.

3. Ozawa E. From dystrophinopathy to sareoglycanopathy: Evolution of a concept of muscular dystrophy. Muscle Nerve 1998;21:421-38.

4. Khadilkar SV, Singh RK, Katrak SM. Sareoglyeanopathies: A report of 25 cases Neurol India 2002;50:27-32.

5. Sharma MC, Mannan R, Singh NG, Gulati S, Kalra V, Sarkar C. Sarcoglycanopathies: An enigmatic form of muscular dystrophy- A report of 7 cases. Neurol India 2004;52:446-9.

6. Pradhan S. New clinical sign in Duchenne muscular dystrophy. Pediatr Neurol 1994;11:298-300.

7. van der Kooi A.J, Ginjaar HB, Busch HF, Wokke JH, Barth PG, de Visser M. Limb girdle muscular dystrophy: A pathological and immunohistochemical re-evaluation. Muscle Nerve 1998;21:584-90.

8. van der Kooi AJ, Barth PG, Busch HF, de Haan R, Ginjaar HB, van Essen AJ, et al. The clinical spectrum of limb girdle muscular dystrophy. A survey in The Netherlands. Brain 1996;119:1471-80.

9. Urtasun M, Saenz A, Roudaut C, Poza JJ, Urtizberea JA, Cobo AM, et al. Limb-girdle muscular dystrophy in Guipuzcoa (Basque Country, Spain), Brain 1998;121:1735-47.

10. Eymard B, Romero NB, Letureq F, Piccolo F, Carrie A, Jeanpierre M, et al. Primary adhalinopathy (sareoglycanopathy): Clinical, pathologic and genetic correlation in 20 patients with autosomal recessive muscular dystrophy. Neurology 1997;48:1227-34.

11. Duggan DJ, Hoffman EP. Autosomal recessive muscular dystrophy and mutations of the sarcoglycan complex. Neuromusc Disord $1996 ; 6: 475-82$.

12. Jeanpierre M, Carrie A, Piccolo F, Letureq F, Azibi K, De Toma C, et al. From adhalinopathies to alpha-sarcoglycanopathies: An overview. Neuromuse Disord 1996;6:463-5.

13. Ben Hamida M, Ben Hamida C, Zouari M, Belal S, Hentati F. Limb-girdle muscular dystrophy 2C: Clinical aspects. Neuromusc Disord 1996;6:493-4.

14. Dincer P, Letureq F, Richard I, Piccolo F, Yalnizoglu D, de Toma C, et al. A biochemical, genetic and elinical survey of autosomal recessive limb girdle muscular dystrophies in Turkey. Ann Neurol $1997 ; 42: 222-9$

15. Moore SA, Shilling CJ, Westra S, Wall C, Wicklund MP, Stolle C, et al. Limb-girdle muscular dystrophy in the United States. J Neuropathol Exp Neurol 2006;65:995-1003.

16. Passos-Bueno MR, Moreira ES, Vainzof M, Marie SK, Zatz M. Linkage analysis in autosomal recessive limb-girdle muscular dystrophy (AR limb girdle muscular dystrophy) maps a sixth form to 5q33-34 (LGMD2F) and indicates that there is at least one more subtype of AR limb girdle muscular dystrophy. Hum Mol Genet 1996;5:815-20

17. Beckmann JS, Brown RH, Muntoni F, Urtizberea A, Bonnemann C, Bushby KM. $66^{\text {th }} / 67^{\text {th }}$ ENMC sponsored international workshop: The limb-girdle muscular dystrophies, 26-28 March 1999, Naarden, The Netherlands. Neuromuscul Disord 1999;9:436-45.

18. Melacini P, Fanin M, Duggan DJ, Freda MP, Berardinelli A, Danieli GA, et al. Heart involvement in muscular dystrophies due to sarcoglycan gene mutations. Muscle Nerve 1999;22:473-9.

19. Politano L, Nigro V, Passamanoaa AL, Petretta V, Comi LI, Papparella $\mathrm{S}$, et al. Evaluation of cardiac and respiratory involvement in sarcoglycanopathies. Neuromuscul Disord 2001;11:178-85.

20. Passos Bueno MR, Vainzof M, Moreira ES, Zatz M. The seven autosomal recessive limb girdle muscular dystrophies (LGMD) in the Brazilian population from LGMD $2 \mathrm{~A}$ to LGMD 2G. Am J Med Genet 1999;82:392-8

Accepted on 16-03-2007

Source of Support: Nil, Conflict of Interest: None declared. 